\title{
E44 DIRECT CONDITIONING OF FINE-SCALE FACIES MODELS TO DYNAMIC DATA BY COMBINING GRADUAL DEFORMATION AND NUMERICAL UPSCALING TECHNIQUES
}

T. SCHAAF ${ }^{1}$, M. MEZGHANI' AND G. CHAVENT ${ }^{2}$

1 Institut Français du Pétrole, 1 et 4, avenue de Bois-Préau, 92852 Rueil Malmaison Cedex - France 2 Ceremade, Université Paris-Dauphine, Place du Maréchal De Lattre de Tassigny, 75775 Paris Cedex 16 and Inria-Rocquencourt, 78153 Le Chesnay Cedex - France

\begin{abstract}
Updating reservoir models through dynamic data integration requires the solution of an inverse problem that may be computationally intensive for multi-million cells geological models. History matching is commonly performed using upscaled flow simulation models without updating the original fine grid geological model. In this way, the drawback is that the consistency with the geostatistical parameters is no longer ensured.

In this paper, we present a new methodology to directly update fine scale geostatistically-based facies models by combining the gradual deformation method for the fine scale model parameterization and numerical upscaling techniques for the coarse scale flow simulation model. Gradual deformation ensures the preservation of the overall geostatistical properties of the fine scale model while upscaling is used as a mandatory step to speed up the simulation process. We used the gradient method to compute the sensitivity coefficients of the flow simulation results with respect to the coefficients of the gradual deformation. This method requires the petrophysical properties (in this case permeability) to be derivable with respect to these coefficients. When considering facies models, the truncation process results in discontinuous changes in the model properties and these derivatives cannot be determined. In order to calculate them, we have introduced the concept of transition facies (in addition to the real petrophysical facies) which appears to modify only slightly the upscaled permeability field.
\end{abstract}

We have successfully applied this methodology to integrate interference test data into a 3D geostatistical model containing about two million cells. This validation highlights the capability of this methodology to update directly geostatistical models in a consistent and efficient way.

\section{Introduction}

Thanks to owerful geostatistical simulation algorithms (e.g. [1]), petroleum geoscientists are currently working on multi-million cells geological models which allow to get a better characterization of the reservoir and to run risk analysis. Hereafter, the main challenge is the proper integration of available data (such as well test and production data) into the reservoir model without losing the geostatistical consistency and with a reasonable computation time. To reach this goal, we applied a new methodology [2] that updates at the same time (through data integration) both upscaled and associated fine-scale (i.e. geostatistical) models.

In this paper, we focus on facies based models which are widely used in reservoir characterization. 
Throughout the paper, lowercase variables refer to the geostatistical scale (e.g. $k$ corresponds to the permeability distribution at the geostatistical scale) while uppercase variables refer to the fluid flow simulation scale (e.g. $K$ corresponds to the permeability field of the fluid flow simulation model).

\section{Parameterization with the gradual deformation methodology}

Gradual deformation of facies models consists in deforming the underlying continuous Gaussian simulation [3]. Let consider a stationary Gaussian random function $Z(x)$ with zero mean and unit variance. The gradual deformation methodology consists in writing a new realization $z$ of the random field $Z$ as a linear combination of $n$ independent realizations $z_{i}$ of $Z$ :

$$
z(\alpha)=\sum_{i=1}^{n} \alpha_{i} z_{i} \text { with }\left\{\begin{array}{l}
\alpha_{i} \in[-1,1], \mathrm{i}=1, \ldots, n \\
\sum_{i=1}^{n} \alpha_{i}^{2}=1
\end{array}\right.
$$

The normality constraint $\sum_{i=1}^{n} \alpha_{i}^{2}=1$ is automatically satisfied when spherical coordinates $\left\{\theta_{1}, \ldots, \theta_{n-1}\right\}$ are used :

$$
z(\theta)=\prod_{i=1}^{n-1} \cos \left(\theta_{i} \pi\right) z_{1}+\sum_{i=1}^{n-1} \sin \left(\theta_{i} \pi\right) \prod_{j=i+1}^{n-1} \cos \left(\theta_{j} \pi\right) z_{i+1}
$$

Hereafter, the new realization $z$ is a function of $n-1$ independent parameters $\left\{\theta_{1}, \ldots, \theta_{n-1}\right\}$.

This algorithm has at least three advantages : (i) preservation of the spatial variability; (ii) reduction of a high dimensional optimization problem to a low dimensional one; (iii) smooth variations in the objective function. Several extensions of this algorithm (e.g. local and structural deformations or deformation of non-Gaussian simulations) are presented in respectively references [1] and [4].

\section{Facies Models generation}

\section{Truncated Gaussian models}

This method consists in truncating a continuous Gaussian simulation with multiple thresholds to represent the distribution of (litho-)facies in a heterogeneous reservoir [5]. It has been proven to be highly flexible for representing a wide range of geological patterns and shapes.

Let $Z(x)(x \in D)$ be a standard Gaussian random function defined over the field $D$ and $I(x)(x \in D)$ an indicator random function defined by truncating $Z(x)$ at threshold $s$ :

$$
I(x)= \begin{cases}0 & Z(x) \leq s \\ 1 & \text { otherwise }\end{cases}
$$

If $Z(x)$ is stationary, then $I(x)$ is also stationary. Equation (3) can be easily extended to the case of several thresholds in order to build models with several facies.

An alternative approach (Fig. 1) consists in truncating the uniform distribution $U(x)$ obtained by applying to $Z(x)$ the repartition function of the Gaussian variable. In that case, thresholds $t_{i}(x)$ are functions of the local proportions of facies $p_{i}(x)$ and the model is generated according to : 


$$
k(x)= \begin{cases}k_{1} \text { if } u(x) \leq t_{1}(x) & , \mathrm{i}=1 \\
k_{i} \text { if } t_{i-1}(x)<u(x) \leq t_{i}(x) & , \mathrm{i}=2, \ldots, N-1 \text { with }\left\{\begin{array}{l}
t_{i}(x)=\sum_{j=1}^{i} p_{j}(x), x \in D \\
k_{N}=\text { Number of facies } \\
\sum_{\mathrm{j}=1}^{N} p_{j}(x)=1, \forall x
\end{array}\right. \text {, otherwise }\end{cases}
$$

To generate a $N$ facies model, one has to specify $(N-1)$ thresholds $t_{i}(x)$. Both approaches result in the same facies model. But conditioning truncated Gaussian simulation models to hard data is currently easier with the second approach (equation (4)) when using the gradual deformation method. This approach has been used for the scope of this work.
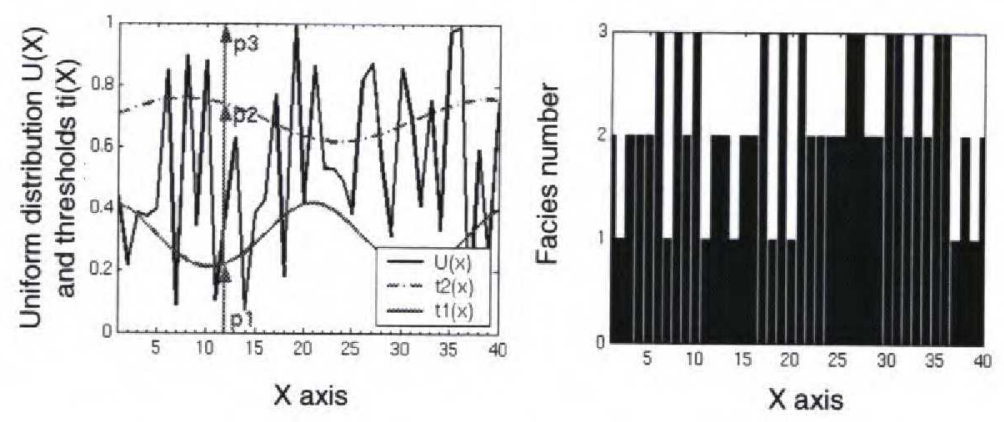

Figure 1 - Generation of a 1D three facies model from a truncated uniform distribution.

It is important to notice that the above procedure is not differentiable because of the truncation process. This will lead us to "smooth" slightly this process in order to permit the use of gradient optimization techniques.

\section{Conditioning to hard data}

Conditioning a point of the model to a specified facies $i$ consists in setting the proportion of this facies $i$ to one (all others proportions being set to zero). This binary case may be compared to the indicator approach [6]. Thus, once all the conditioning points are specified, thresholds are obtained throughout the model by indicator kriging with proper specifications.

\section{Numerical upscaling technique}

We used an upscaling technique that solves the pressure field at the geostatistical scale i.e. for all the $N_{x} \times N_{y} \times N_{z}$ cells of the upscaled block. We considered "permeameter" boundary conditions. This technique assumes that Darcy's law exists at the scale of the upscaled block. Thus, writing the Darcy's law, we get for the equivalent permeability (in the X direction) :

$$
K_{e q}=\frac{1}{N_{x}-1} \sum_{I=1}^{N_{x}-1}
$$
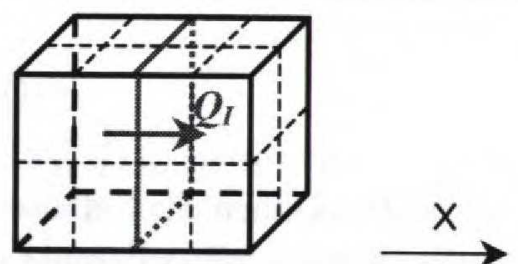

where $S$ is the area of the section perpendicular to the $\mathrm{X}$ direction, $Q_{I}$ the flux along the $\mathrm{X}$ direction at the block scale and $\Delta P$ the pressure difference between the two faces perpendicular to the $\mathrm{X}$ axis and distant of $N_{x} \Delta x$. The flux $Q_{I}$ is equal to the sum of the local fluxes at the geostatistical scale. For a cubic mesh at this scale, we get : 


$$
Q_{I}=\sum_{J=1}^{N_{y}} \sum_{L=1}^{N_{z}} t_{I+1 / 2, J, L}\left(p_{I+1}-p_{I}\right)
$$

where $t_{I+1 / 2, J, L}$ represents the transmissivity between cells $(I, J, L)$ and $(I+1, J, L)$ and $p_{I}$ the pressure of the cell $(I, J, L)$. Equivalent permeability formula for the $\mathrm{Y}$ and $\mathrm{Z}$ directions are straightforward.

\section{Principle of the methodology}

This methodology can be seen as a coupling of the inversion and upscaling steps of the data integration process, in a way which ensures the consistency of the upscaled model with the geostatistical parameters.

The upscaling is now part of the parameterization. It is no longer a pre-processing step for the fluid flow simulator. Updating reservoir models through dynamic data integration requires then the sensitivity coefficients of the flow simulation results (i.e. pressures $P$, as we consider a monophasic case) with respect to the coefficients $\theta_{i}$ of the gradual deformation (i.e. the parameterization of the fine scale model). We calculate these sensitivity coefficients $\partial P / \partial \theta_{i}$ using the gradient method ([7],[8]). Derivation of the flow equations with respect to $\theta_{i}$ requires then the knowledge of $\partial K / \partial \theta_{i}$, and consequently the derivation of the upscaling, truncation and gradual deformation steps.

\section{Flowchart of the direct problem}

All the preceding steps are summarized in figure 2 . The gradients of the petrophysical properties (in this case permeability) with respect to the coefficients of gradual deformation are not defined, because of the truncation process. As seen on figure 2, this process (step 3) results in discontinuous changes in the model properties.

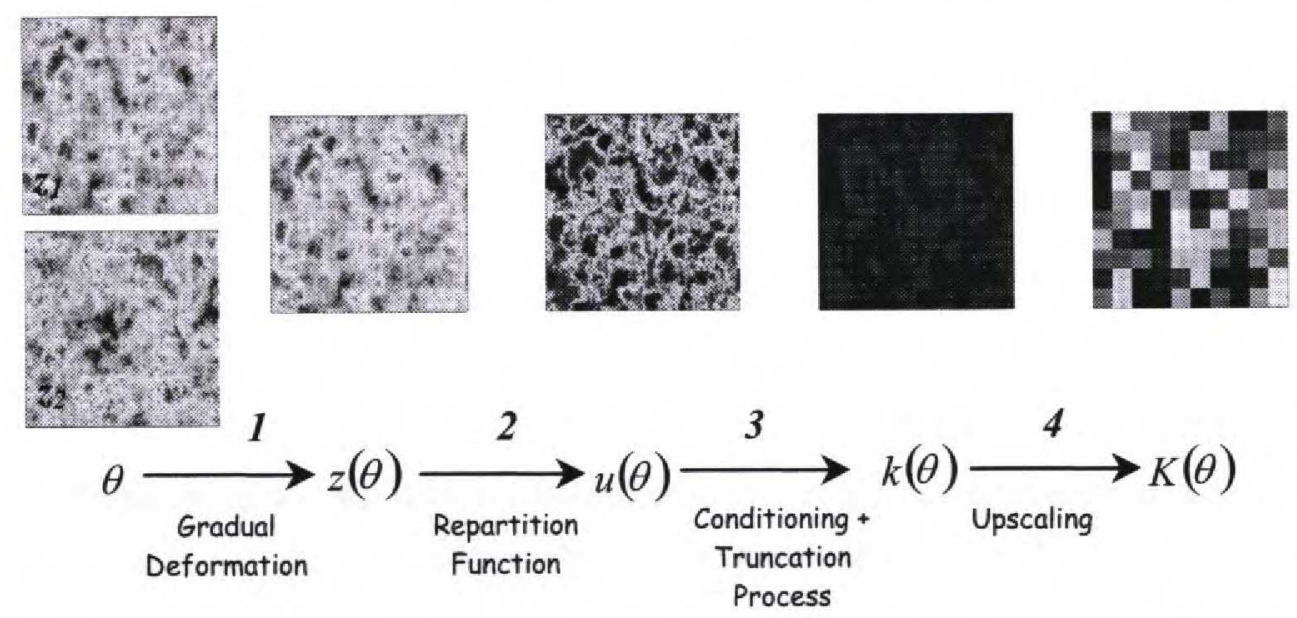

Figure 2 - Flowchart of the direct problem

From the derivation of equations (5) and (6) with respect to coefficient $\theta_{i}$, we get the sensitivity distribution $\partial K / \partial \theta_{i}$. Two series of terms appear in this derivation :

1) Terms $\left\{t_{I+1 / 2, J, L}, p_{I+1}, p_{I}\right\}$ which are already computed through the upscaling process, 
2) Terms $\left\{\frac{\partial t_{I+1 / 2, J, L}}{\partial \theta_{i}}, \frac{\partial p_{I+1}}{\partial \theta_{i}}, \frac{\partial p_{I}}{\partial \theta_{i}}\right\}$ which have to be determined.

Terms like $\partial p_{I} / \partial \theta_{i}$ are determined through the derivation of the linear system solved by the numerical upscaling technique. Terms like $\partial t / \partial \theta_{i}$ are determined through the derivation of the formula used for the transmissivity computation (in this case harmonic mean) which itself needs $\partial k / \partial \theta_{i}$ derivatives.

From a mathematical point of view, the function $k=f(u(\theta))$ is a step function which derivative is a Dirac. We have introduced the concept of transition facies to ensure the existence of the gradient $\partial k / \partial \theta_{i}$ and so of the derivative :

$$
\frac{\partial K_{j}}{\partial \theta_{i}}=\sum_{l=1}^{N t} \frac{\partial K_{j}}{\partial k_{l}} \frac{\partial k_{l}}{\partial \theta_{i}} \text { with } N t=\text { Number of cells of the block } j
$$

Concept of transition facies to ensure the derivability of the truncation step

For a model with $N$ real petrophysical facies (cf. equation (4)), we introduce $(N-1)$ transition facies (one between each real facies) with fixed percentage. We end up with $N^{*}=(2 N-1)$ real and transition facies of percentages $p_{i}^{*}\left(u^{l}\right)$ which correspond to $2(N-1)$ thresholds $t_{i}^{*}\left(u^{l}\right)$. For a point $u^{l}$ belonging to a transition facies interval, permeability is computed by cubic spline interpolation with endpoint values equal to the permeability of the two surrounding real facies :

$$
k\left(u^{l}\right)=\left\{\begin{array}{lll}
k_{1} & \text { if } u^{l} \leq t_{1}^{*}\left(u^{l}\right) & , \mathrm{i}=1 \\
k_{(i+1) / 2} & \text { if } t_{i-1}^{*}\left(u^{l}\right)<u^{l} \leq t_{i}^{*}\left(u^{l}\right) & , \text { i odd, } \mathrm{i} \neq N^{*} \\
s_{i / 2} & \text { if } t_{i-1}^{*}\left(u^{l}\right)<u^{l} \leq t_{i}^{*}\left(u^{l}\right) & , \text { i even, } \mathrm{i} \neq N^{*} \\
k_{N} & \text { otherwise }
\end{array} \text { with } t_{i}^{*}\left(u^{l}\right)=\sum_{j=1}^{i} p_{j}^{*}\left(u^{l}\right)\right.
$$

where $t_{i}^{*}\left(u^{l}\right)$ is the threshold number $i$ at the point $u^{l}$ of the uniform distribution and $s_{i / 2}$ the cubic spline.

From figure 2, we see that the gradient $\frac{\partial k_{l}}{\partial \theta_{i}}$ can be expressed as : $\frac{\partial k_{l}}{\partial \theta_{i}}=\underbrace{\frac{\partial k_{l}}{\partial u^{l}}} \underbrace{\frac{\partial u^{l}}{\partial z^{l}}} \underbrace{\frac{\partial z^{l}}{\partial \theta_{i}}}$

We have analytical expressions for all three gradients :

1) Term 1 is equal to zero if $u^{l}$ corresponds to a real facies or is equal to the spline derivative $s_{i / 2}^{\prime}$ if $u^{\prime}$ corresponds to a transition facies,

2) Term 2 is the derivative of the repartition function which is the probability density function at value $z^{l}$

3) Term 3 is the derivative of equation (2).

Thus, we are able to compute both gradients $\frac{\partial k_{l}}{\partial \theta_{i}}$ and hence $\frac{\partial K_{j}}{\partial \theta_{i}}$ using equation (7). This calculation is done for each diagonal term of the permeability tensor. 


\section{Interference test case}

The objective is to match synthetic pressure data simulated with a reference fine-scale facies model using the gradual deformation method.

\section{Synthetic reservoir description}

The synthetic model illustrated in figure 3 is a $3 \mathrm{D}$ reservoir containing $201 \times 201 \times 50$ cells of dimension $(\Delta \mathrm{x}=20 \mathrm{~m}, \Delta \mathrm{y}=20 \mathrm{~m}, \Delta \mathrm{z}=1 \mathrm{~m})$. This model includes three facies which permeabilities are respectively 10 $\mathrm{mD}, 100 \mathrm{mD}$ and $1500 \mathrm{mD}$ and percentages respectively $20 \%, 50 \%$ and $30 \%$. The other petrophysical properties are also constant and known : porosity is $30 \%$ and total compressibility is $1.10^{-4}$ bar $^{-1}$. The variogram is spherical with anisotropic correlation lengths $\left(l_{\mathrm{x}}=1000\right.$ $\mathrm{m}, \mathrm{l}_{\mathrm{y}}=200 \mathrm{~m}$ and $\mathrm{l}_{\mathrm{z}}=6 \mathrm{~m}$ ). At the geostatistical scale, we assume $k_{x}=k_{y}$ and $k_{z} / k_{x}=0.1$. The fluid flow simulation is single phase with a viscosity value of 1.1 cP. A production well (noted $\mathrm{P}_{1}$ ) with a radius of 7.85 $\mathrm{cm}$ and no skin is located at the center of the reservoir and perforated over the 30 upper layers. Its history comprises a period of constant production rate at 600 $\mathrm{m}^{3} /$ day during seven days and a buildup period during

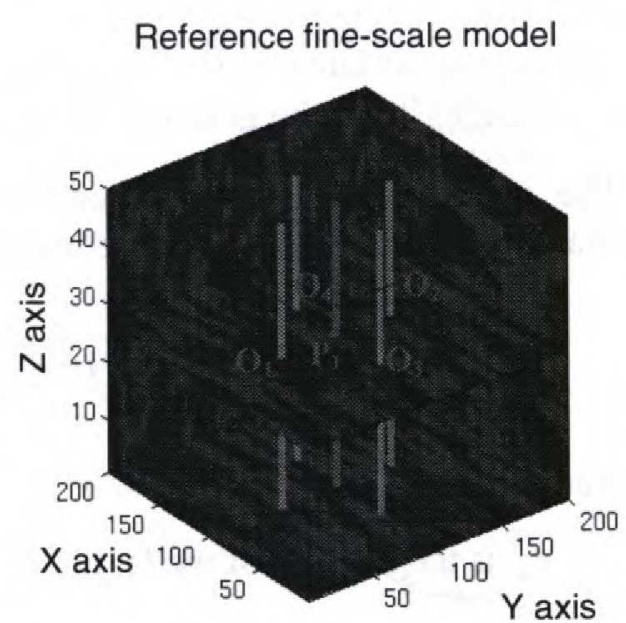

Figure 3 - Reference fine-scale facies model 55 days. Four observation wells (noted $\mathrm{O}_{1}, \mathrm{O}_{2}, \mathrm{O}_{3}$ and $\mathrm{O}_{4}$ ) are located around $\mathrm{P}_{1}$ in a diamond pattern. These wells are perforated over the 30 lower layers.

\section{Reference and initial data}

For a given synthetic fine-scale model, we compute the synthetic pressure for the five wells using an in-house monophasic flow simulator. The interference test data include the bottom-hole pressure evolution at the production and observation wells (Fig. 6) and the pressure derivative evolution at the production well.

For the inversion process, the upscaling step results in a flow simulation model composed of :

1) 43 blocks along the $X$ axis with variable $\Delta X$,

2) 43 blocks along the $Y$ axis with variable $\Delta Y$,

3) 10 blocks along the $Z$ axis with $\Delta Z=5 \mathrm{~m}$.

At each well location, a local refinement is used along $\mathrm{X}$ and $\mathrm{Y}$ directions, and the grid block dimensions correspond to the fine scale model $(\Delta X=\Delta x=20 \mathrm{~m}$ and $\Delta Y=\Delta y=20 \mathrm{~m})$. This simulation model contains 18490 blocks. It corresponds to a reduction of around $99 \%$ of the initial number of cells.

\section{Impact of transition facies on the simulation permeability field and the simulation results}

Transition facies is an artifice that ensures the derivability of the truncation step. We have conducted sensitivity analysis to quantify its impact on the permeability field of the simulation model and the simulation results themselves. We considered four values : 10, 5, 3 and $1 \%$ attributed to each of the two transition facies (as we have three real facies). For the upscaled 
simulation blocks, the permeab calculated with transition facies can differ slightly from the upscaled value computed without transition facies. This variation depends on the block size and the transition facies proportion. The key point is that pressure variations obtained at the five wells for the different proportion values are very small.

\section{Inverse problem formulation}

As mentioned above, all petrophysical and geostatistical values of the reservoir are assumed to be known. In the same way, all facies properties (permeabilities and proportions) are also assumed to be known. The objective is to characterize the distribution of facies by adjusting the coefficients $\theta_{i}$ of the gradual deformation.

The inversion process is based on a non-linear Powell "Dog-Leg" optimization algorithm. Matching the interference test consists in minimizing the objective function :

$$
F(\theta)=\frac{1}{2 n_{w}} \sum_{i=1}^{n_{i w}} \frac{1}{n_{o b s}^{i}} \sum_{j=1}^{n_{o b s}^{i}}\left(\frac{D_{i j}^{s i m}(\theta)-D_{i j}^{o b s}}{\sigma_{i j}}\right)^{2}
$$

where :

- $n_{w}$ is the number of wells (i.e. $n_{w}=5$ ) and $n_{o b s}^{i}$ is the number of measures at well number $i$,

- $D^{o b s}$ is the measured data and $D^{s i m}(\theta)$ the simulated data,

- $\sigma$ is a weighting coefficient for the measurements.

For the observation wells, both draw-down and buildup pressure variations were considered whereas only the buildup pressures (and associated derivatives) were considered for the production well (Fig. 6).

\section{Pressure and derivative matching}

Pressure and derivative matching was obtained by performing three successive series of optimizations (30 iterations maximum). Each series is associated to a set of seven realizations $z_{i}$ for the gradual deformation (which implies six deformation coefficients $\theta_{i}$ ). For the second and third series, we keep the optimal distribution $z_{\text {opt }}$ of the preceding optimization (Fig. 4). These realizations were independent from the one used to generate the reference data.

As transition facies are an artifice, we have conducted a sensitivity analysis with respect to the percentage attributed to these transition facies. We considered again the four values : 10, 5,3 and $1 \%$ attributed to each of the two transition facies. Figure 5 represents the evolution of the objective function $F$ for these four percentage values. Each series of iterations stops whenever function $F$ variations are too small. For a percentage greater or equal to $5 \%$, the value of the function $F$ drops of nearly two orders of magnitude : optimization succeeded without problems (which is confirmed by another sensitivity analysis conducted on the gradients $\partial K_{j} / \partial \theta_{i}$ validity as a function of the transition percentage). The final values of the function $F$, noted $F_{\min }$, is 57 , 54,192 and 247 for transition percentage of respectively $10,5,3$ and $1 \%$ (initial values are respectively $2616,2455,2406$ and 2362 ).

Figure 6 illustrates the successful pressure and derivative matching with a proportion of 5 percent for each transition facies. We plot three series of data : reference, initial and final data. 
As mentioned above, the transition facies method is an artifice to allow the derivation of the modelisation chain. In order to estimate its impact, we generated the fine-scale model corresponding to the $F_{\min }$ value (and associated deformation coefficients $\theta_{i}$ ) without any transition facies i.e. only with the three real facies with proportions 20,50 and 30 percent respectively. This model was considered as the optimal solution of the optimization process.

Figure 7 illustrates the comparison between the reference pressure data and the pressure solution obtained with the final model at the fine scale.

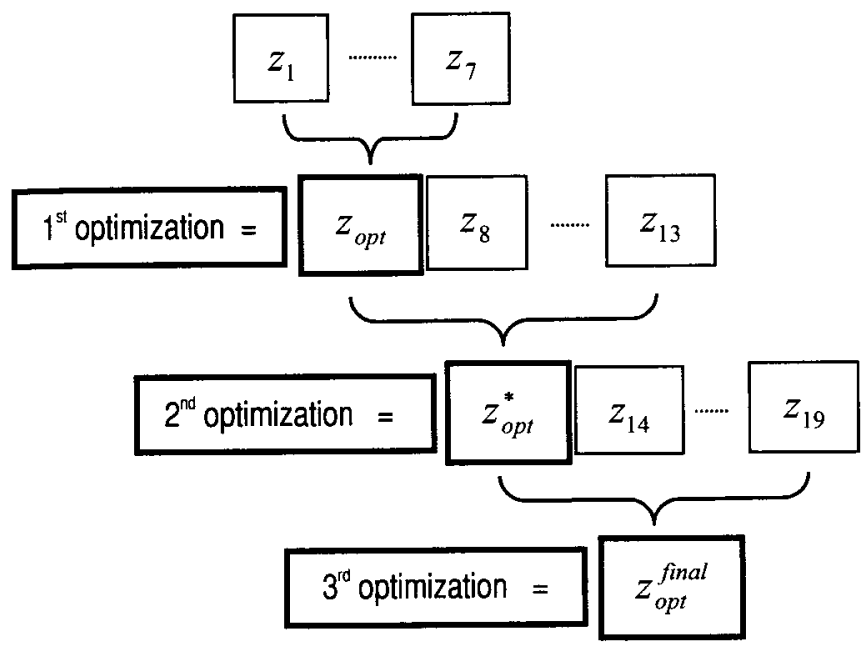

Figure 4 - Optimization strategy

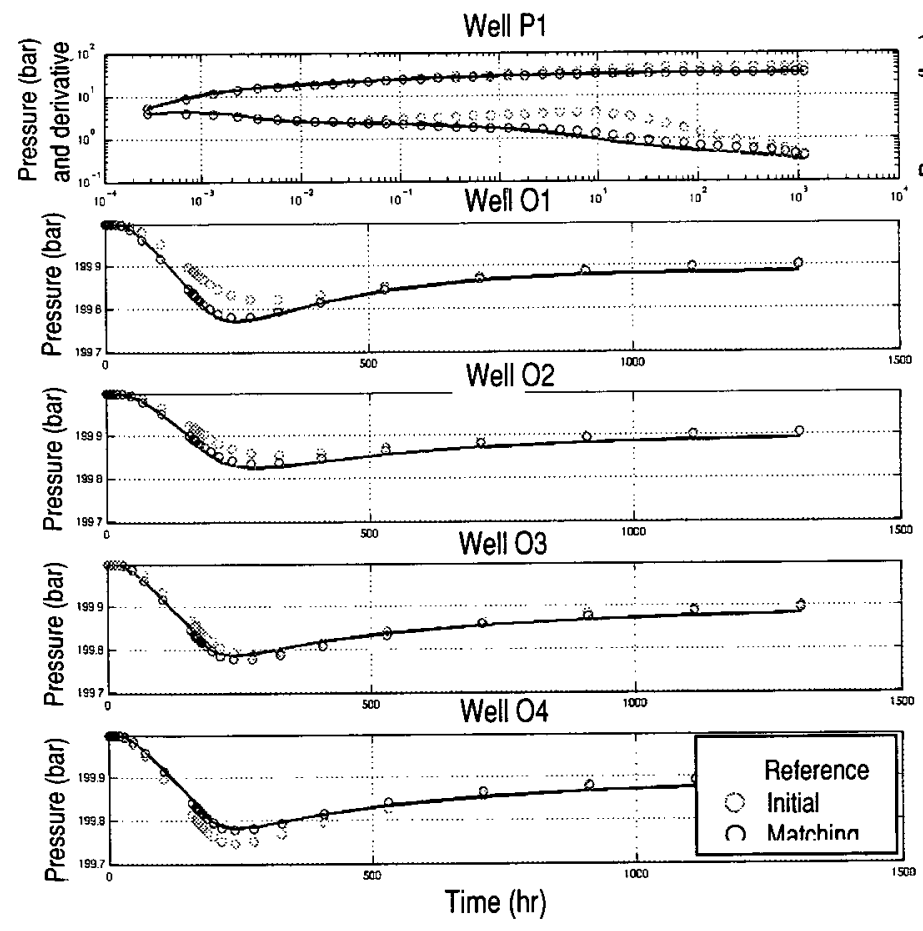

Figure 6 - Pressure and derivative matching in the 5 percent transition facies case

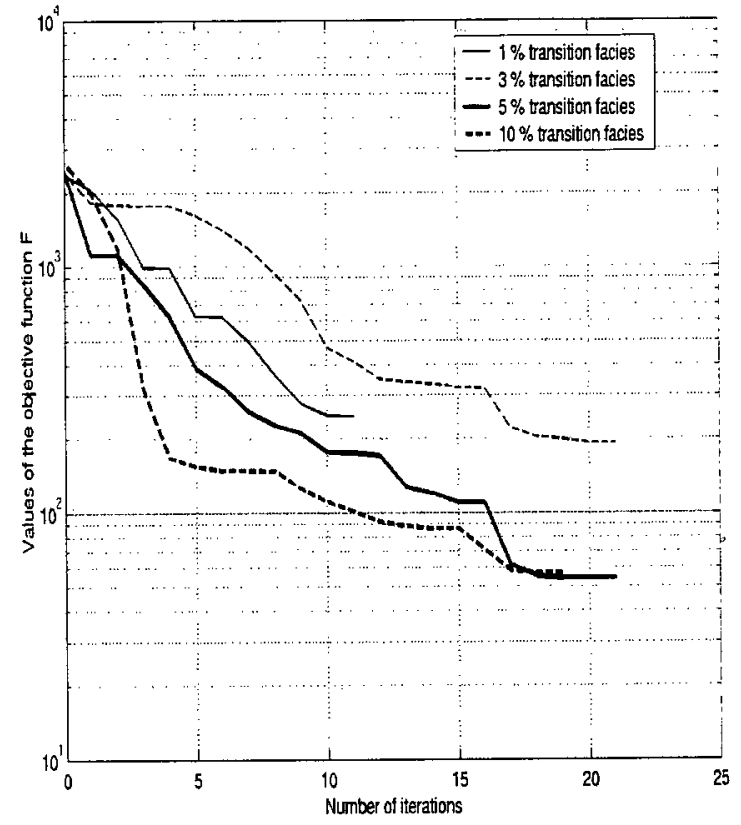

Figure 5 - Evolution of the objective function $F$ for the four transition facies percentages
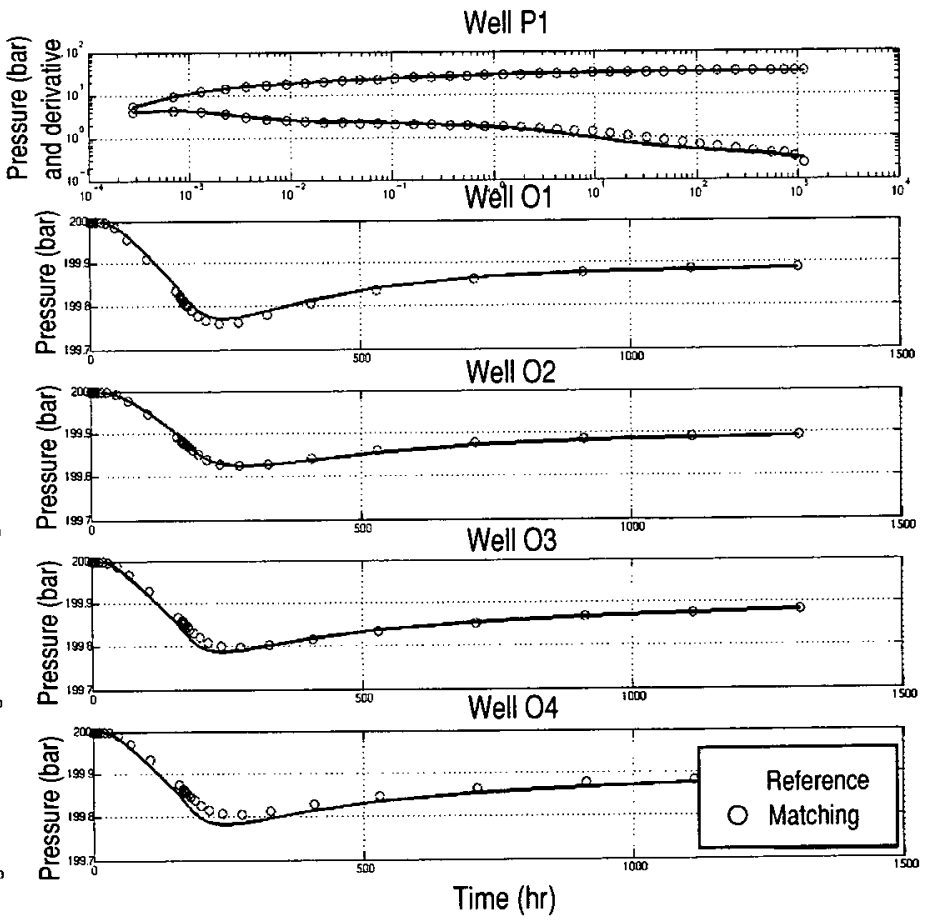

Figure 7 - Pressure and derivative matching of the fine-scale model without transition facies 


\section{Conclusions}

We have successfully applied a new methodology to integrate interference test data into a 3D geostatistical facies based model containing about two million cells. This novel method is based on the computation of gradients with transition facies. It combines the gradual deformation method and upscaling step in an optimization loop to properly integrate available data into facies based models. This validation highlights the capability of this methodology to directly update geostatistical models in a consistent and efficient way.

Acknowlegments

The authors thank the Institut Français du Pétrole for its permission to publish this article. The single phase fluid flow simulation was performed using the WELGEM software. WELGEM was developped in an IFP JIP sponsored by ANADARKO, BP, BHP-Billiton, GDF, PEREZ-COMPANC, PETROBRAS, PHILLIPS and TOTALFINAELF.

\section{References}

[1]Le Ravalec, M., Noetinger, B. \& Hu, L.Y. (2000). "The FFT Moving Average (FFT-MA) generator : an efficient numerical method for generating and conditioning gaussian simulations", Math. Geol. 32(6), 701-723.

[2]Mezghani, M. \& Roggero, F. (2001). "Combining gradual deformation and upscaling techniques for direct conditioning of fine scale reservoir models to dynamic data", SPE 71334.

[3]Roggero, F. \& Hu, L.Y. (1998). "Gradual deformation of continuous geostatistical models for history matching", SPE 49004.

[4]Hu, L.Y. (2000). "Gradual deformation of non-gaussian stochastic simulations" in Kleingeld \& Kridge (eds.), Geostatistics 2000 Cape Town.

[5]Matheron, G., Beucher, H., de Fouquet, C., Galli, A. \& Ravenne, C. (1987). "Conditional simulation of the geometry of fluvio-deltaic reservoirs", SPE 16753.

[6]Deutsch, C.V. \& Journel, A.G. (1992). "GSLIB geostatistical software library and user's guide", Oxford University Press, New York, Oxford, 340 p.

[7]Anterion, F., Eymard, R. \& Karcher, B. (1989). "Use of parameter gradients for reservoir history matching", SPE 18433.

[8]Rahon, D., Blanc, G. \& Guérillot, D. (1996). "Gradients method constrained by geological bodies for history matching", SPE 36568. 
